\title{
Enhanced Optical Output of Near-Ultraviolet Light-Emitting Diodes by a Monolayer of Nanospheres
}

\author{
Wei Wan, ${ }^{1,2}$ Zhanxu Chen $\mathbb{D}^{1},{ }^{1}$ Yongzhu Chen, ${ }^{1}$ and Gengyan Chen ${ }^{1}$ \\ ${ }^{1}$ School of Optoelectronic Engineering, Guangdong Polytechnic Normal University, Guangzhou 510665, China \\ ${ }^{2}$ State Key Laboratory of Optoelectronic Materials and Technologies, School of Physics, Sun Yat-sen University, \\ Guangzhou 510275, China
}

Correspondence should be addressed to Zhanxu Chen; chenzhanxu78@126.com

Received 30 September 2019; Revised 4 February 2020; Accepted 15 February 2020; Published 20 April 2020

Academic Editor: Sergio Bietti

Copyright (C) 2020 Wei Wan et al. This is an open access article distributed under the Creative Commons Attribution License, which permits unrestricted use, distribution, and reproduction in any medium, provided the original work is properly cited.

The optical output of near-ultraviolet (NUV) light-emitting diodes (LEDs) was improved by including a monolayer of hexagonal close-packed polystyrene (PS) nanospheres. PS nanospheres with different sizes were deposited on the indium tin oxide layer of the NUV LEDs. The electroluminescence results showed that the light extraction efficiency of the NUV LEDs was increased by the inclusion of PS nanospheres, and the maximum optical output enhancement was obtained when the size of the nanospheres was close to the light wavelength. The largest enhancement of the optical output of 1.27-fold was obtained at an injection current of $100 \mathrm{~mA}$. The enhanced optical output was attributed to part of the incident light beyond the critical angle being extracted when the exit surface of the NUV LEDs had a PS nanosphere monolayer. This method may serve as a low-cost and effective approach to raise the efficiency of NUV LEDs.

\section{Introduction}

Near-ultraviolet (NUV) light-emitting diodes (LEDs) have been used in various applications such as biological agent identification, ultraviolet curing, fake bill detection, and solid-state lighting [1-3]. Although gallium nitride- (GaN-) based NUV LEDs are now being produced commercially, their capacities still need to be improved. For example, the external quantum efficiency (EQE) of NUV LEDs is still unsatisfactorily low [4]. One of the main causes of the low EQE of NUV LEDs is their low light extraction efficiency (LEE), which is caused by the total internal reflection by the refractive index difference between the semiconductor and air [5]. Various approaches have been used to enhance the LEE of NUV LEDs, such as surface plasmon enhancement [6-8], surface roughening [9-11], aluminum (Al) nelectrodes [12], periodic hole patterns [13], additional silver nanostructures [14-17], and Al-doped indium tin oxide (ITO) electrodes [18].

Arrays of periodic nanospheres exhibit unique scattering properties because of the Mie resonance of individual spheres and Bragg diffraction arising from the periodic arrangement of the nanospheres [19]. Various types of nanospheres have been used to enhance the LEE of blue LEDs, and the outstanding enhancement has been achieved [20-27]. This approach has advantages of low cost, high throughput, and large area [21]. Additionally, this approach can avoid degrading the electrical properties of the devices because it does not involve etching or lithographic processing [27]. However, the design and fabrication of nanospheres on the ITO layer of commercial NUV LEDs have not been studied. It is very important to determine the optimal size of nanospheres for specific-wavelength NUV LEDs.

In this paper, we study the optical output enhancement of a monolayer of hexagonal close-packed polystyrene (PS) nanospheres adsorbed on the ITO layer of NUV LEDs. To investigate the effect of PS nanosphere size on the optical properties of the NUV LEDs, monolayers of nanospheres with different sizes are prepared on the NUV LEDs. PS nanospheres with diameters of $250,300,400,600,800,1000,1200$, and $1500 \mathrm{~nm}$ are investigated. The electroluminescence results show that a PS nanosphere monolayer increases the 
optical output compared to that of the corresponding LED without PS nanospheres. The maximum optical output enhancement is achieved using PS nanospheres with a diameter of $400 \mathrm{~nm}$. The light output power of the LEDs with a monolayer of $400 \mathrm{~nm}$ PS nanospheres increased by $127 \%$ at an injection current of $100 \mathrm{~mA}$ compared with that of the corresponding LED without a nanosphere monolayer. The experimental results are confirmed by a three-dimensional (3D) finite difference time-domain (FDTD) simulation. The improvement of NUV LED performance and the physical mechanism of light extraction are discussed in detail.

\section{Experimental Methods}

Figure 1 shows a schematic diagram of an NUV LED with a monolayer of hexagonal close-packed PS nanospheres. The planar NUV (385 nm) AlGaN/InGaN multiple quantum well (MQW) LED structure was grown on a 2-inch sapphire substrate by metal-organic chemical vapor deposition. The LED structures consisted of a sapphire substrate, $2 \mu \mathrm{m}$ thick undoped GaN layer, $3 \mu \mathrm{m}$ thick n-GaN layer, $300 \mathrm{~nm}$ thick five-period $\mathrm{AlGaN} / \mathrm{InGaN} \mathrm{MQW}$ active layer, and $100 \mathrm{~nm}$ thick p-GaN layer. The device surface was a transparent ITO electrode, which acted as a current spreading layer. LEDs with dimensions of $185 \times 275 \mu \mathrm{m}$ were formed by mesa etching of the exposed n-type $\mathrm{GaN}$ via standard photolithography, ITO wet etching, and subsequent inductively coupled plasma etching. $\mathrm{Cr} / \mathrm{Pt} / \mathrm{Au}$ was deposited on top of the ITO surface as well as the exposed n-GaN layer as contacts for both the $\mathrm{p}-\mathrm{GaN}$ and $\mathrm{n}-\mathrm{GaN}$ layers. Nanosphere monolayer arrays were formed on top of the ITO surface of NUV LEDs by self-assembly $[28,29]$. To study the influence of nanosphere size on the LEE of NUV LEDs, we fabricated monolayers of PS nanospheres with different diameters on the ITO surface of the LEDs. All of the devices were fabricated from the same LED wafer to eliminate the variations from wafer to wafer and ensure the reliability of the results. The light output power of the LED chip was measured using an integrating sphere.

\section{Results and Discussion}

Figure 2 shows scanning electron microscopy (SEM) images of monolayers of PS nanospheres with different sizes. The size and distribution of PS nanospheres were relatively uniform. Apart from a few defects, the PS nanospheres were closely arranged in a single layer to form a hexagonal closepacked periodic structure. In experiments, a 2-inch LED wafer was divided into eight parts to prepare the samples. Sample A was a conventional LED without a PS nanosphere monolayer, which acted as a reference device. Samples B, C, D, E, F, G, H, and I were coated with monolayers of PS nanospheres with diameters of 250, 300, 400, 600,800, 1000, 1200, and $1500 \mathrm{~nm}$, respectively. The morphologies of the PS nanosphere monolayers in samples B, C, D, E, F, G, H, and I are shown in Figures $2(\mathrm{a})-2(\mathrm{~h})$, respectively. The PS nanospheres strongly adhered on the ITO surface.

Figure 3(a) illustrates the injection current-light output $(I-L)$ characteristics of NUV LEDs with and without a

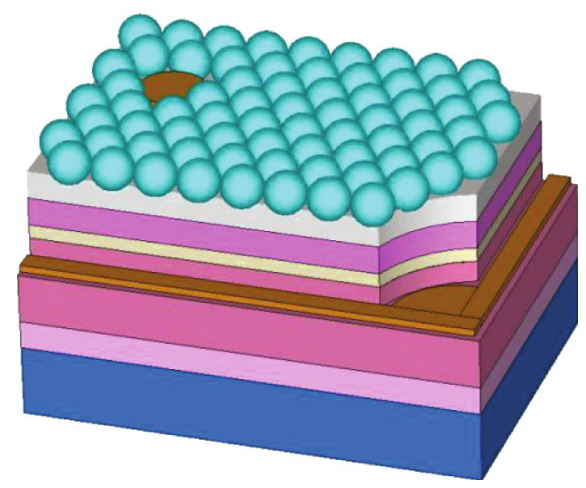

FIgURE 1: Schematic diagram of the structure of an NUV LED with a monolayer of hexagonal close-packed PS nanospheres.

monolayer of hexagonal close-packed PS nanospheres. The optical output power at the same injection current increased upon including a monolayer of PS nanospheres. The optical output power of samples B, C, D, E, F, G, H, and I increased by $118 \%, 123 \%, 127 \%, 121 \%, 119 \%, 120 \%, 114 \%$, and $110 \%$, respectively, compared with that of the reference sample A at an $I$ of $100 \mathrm{~mA}$. Figure 3(b) shows the injection currentvoltage $(I-V)$ characteristics of the NUV LEDs with and without PS nanosphere monolayers measured at room temperature. The forward voltages of the NUV LEDs with and without PS nanosphere monolayers were almost same $(3.3 \mathrm{~V}$ at $20 \mathrm{~mA})$, and all the $I-V$ curves almost overlapped. Therefore, the deposition of a PS nanosphere monolayer on the LED surface did not markedly degrade the electrical properties of the NUV LEDs.

To verify the experimental results, a 3D FDTD simulation was carried out to investigate the size effect of a hexagonal close-packed PS nanosphere monolayer on the light extraction behavior of the NUV LEDs. The simulated LED structure is shown in Figure 4(a), which consisted of a $1000 \mathrm{~nm}$ sapphire substrate, $5200 \mathrm{~nm}$ GaN layer (including $\mathrm{n}-\mathrm{GaN}, \mathrm{MQW}$, and p-GaN layers), and $300 \mathrm{~nm}$ ITO layer. In the simulation, a dipole as a point light source was used and the wavelength was $385 \mathrm{~nm}$, which corresponded to the center wavelength of the emission spectrum. The refractive indices of GaN, ITO, and PS are approximately $2.745,1.9$, and 1.59, respectively, at a wavelength of $385 \mathrm{~nm}[20,30]$. Monolayers of hexagonal close-packed PS nanospheres with different sizes between 250 and $1500 \mathrm{~nm}$ were investigated in the simulation. In the simulation, perfectly matched layers (PML) absorbing boundary conditions were assumed on all sides of the model. There were about $16 \times 17$ nanospheres in the simulation region. Figure 4(b) shows the calculated and experimental light extraction enhancement ratio of the LEDs as a function of the size of the PS nanospheres. The enhancement ratio is defined as the optical output power of the LEDs coated with monolayers divided by the conventional LED. The trend of the calculated results was consistent with that of the experimental results. The simulated ratios were lower than those determined experimentally, which may be because a plane detector was used in the simulation and an integrating sphere is used in the experiments. And the integrating sphere can collect more light. Both the 


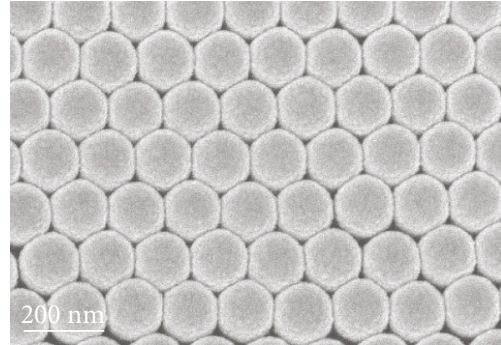

(a)

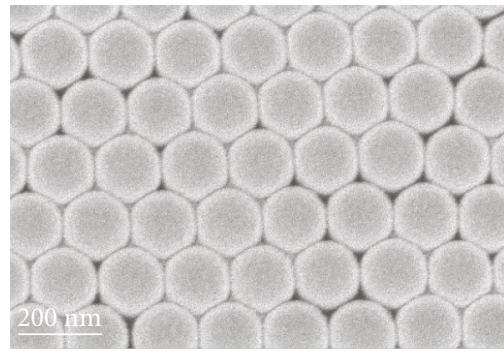

(c)

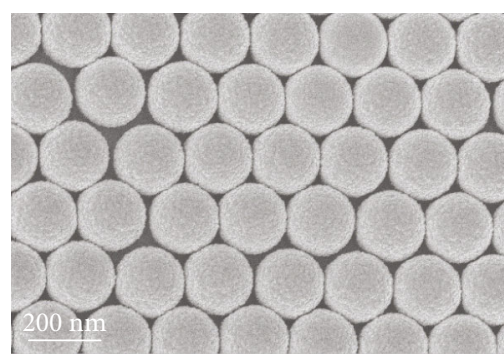

(e)

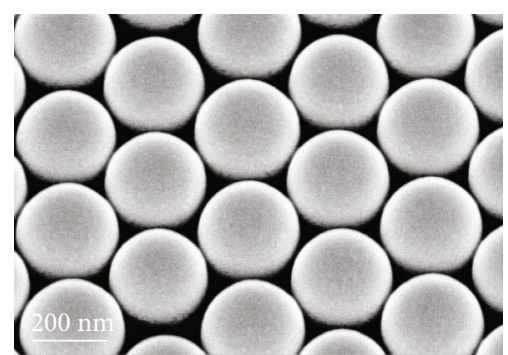

(g)

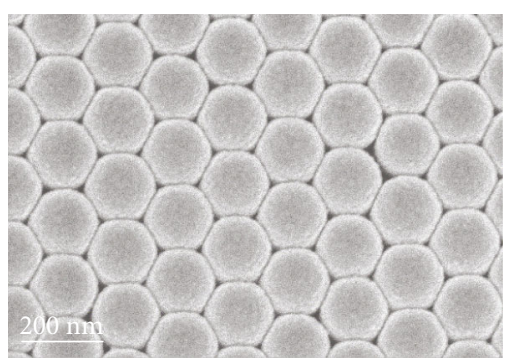

(b)

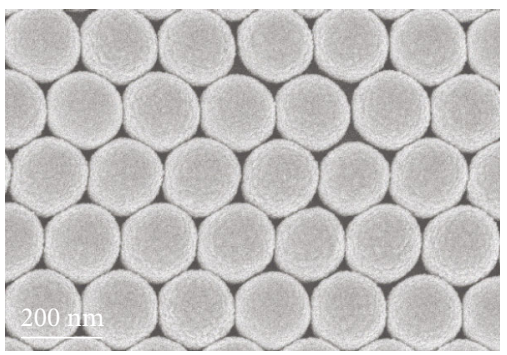

(d)

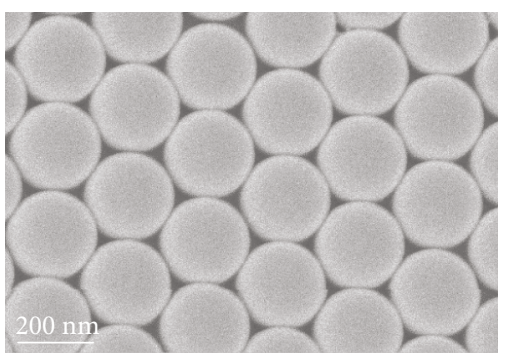

(f)



(h)

Figure 2: SEM images of monolayers of PS nanospheres with sizes of (a) $250 \mathrm{~nm}$, (b) $300 \mathrm{~nm}$, (c) $400 \mathrm{~nm}$, (d) $600 \mathrm{~nm}$, (e) $800 \mathrm{~nm}$, (f) $1000 \mathrm{~nm},(\mathrm{~g}) 1200 \mathrm{~nm}$, and (h) $1500 \mathrm{~nm}$.

experimental and calculated results showed that the optimum extraction efficiency was achieved when the scattering particles had a size close to the wavelength of light, which is consistent with the results in Refs. [27, 31].

The above results show that the formation of a monolayer of hexagonal close-packed PS nanospheres on the ITO surface of LEDs can improve their LEE. To investigate the physical mechanism of the LEE enhancement, the transmission spectrum of the related structures at an emission wavelength of $385 \mathrm{~nm}$ was calculated by FDTD simulation; the results obtained for $\mathrm{s}$ and $\mathrm{p}$ polarization are shown in Figures 5(a) and 5(b), respectively. For the conventional LEDs without a PS nanosphere monolayer, the transmission decreased to zero beyond the critical angle of $21.5^{\circ}$. After being covered with a monolayer of $400 \mathrm{~nm}$ PS nanospheres, two extra transmission peaks appeared at about $23^{\circ}$ and $31^{\circ}$ for both polarizations. This implies that the incident light beyond the critical angle can be partly extracted when the LED is coated with PS nanospheres, leading to enhanced LEE.

It is important to distinguish the physical mechanisms that led to the appearance of the two additional peaks in the transmission spectra. We calculated spatial distributions of electric field intensity of three adjacent spheres corresponding to the two peaks for $\mathrm{p}$ polarization, as shown in Figures 5(c) and 5(d). In the simulation, the Bloch boundary conditions were set around the unit cell and PML absorbing boundary conditions were used at the top and bottom boundaries of the cell. The distribution of electric field 


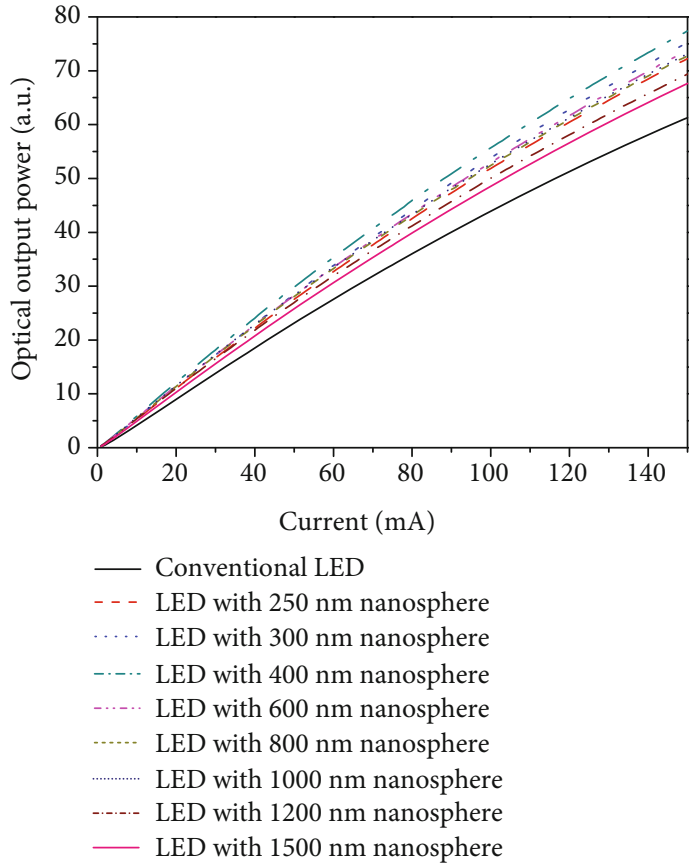

(a)

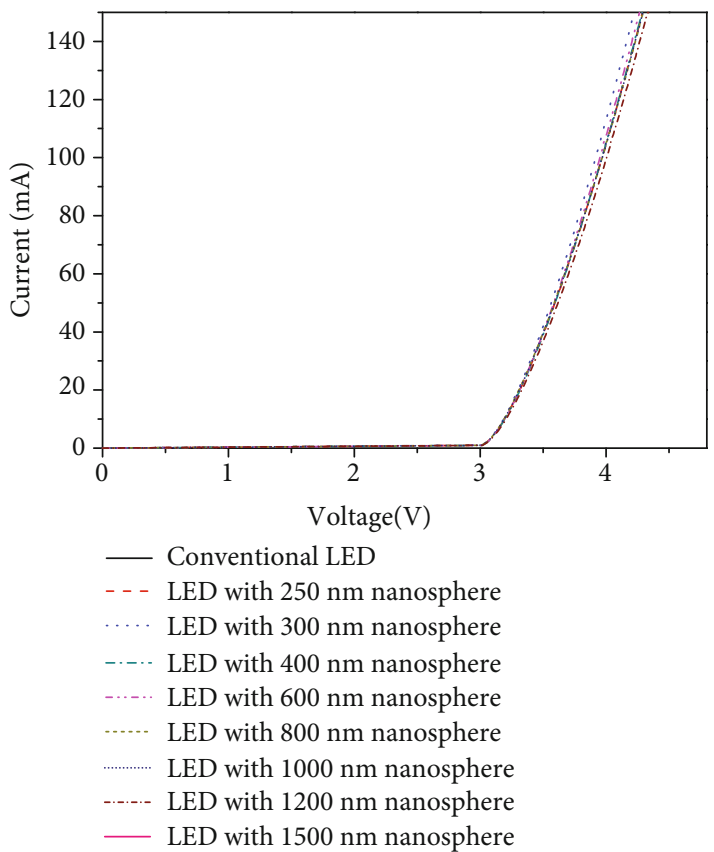

(b)

FIGURE 3: Electroluminescence curves of the NUV LEDs with and without PS nanosphere monolayers. (a) Optical output power versus injection current and (b) injection current versus voltage characteristics.

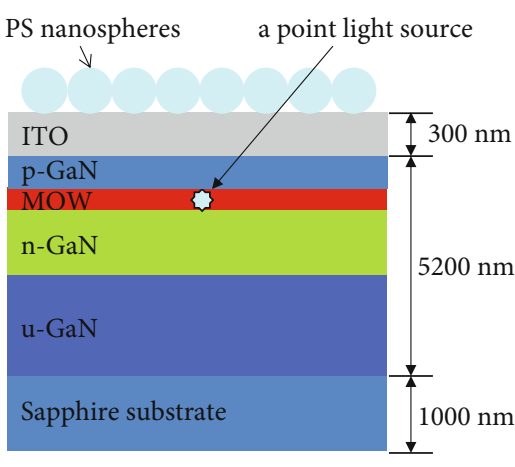

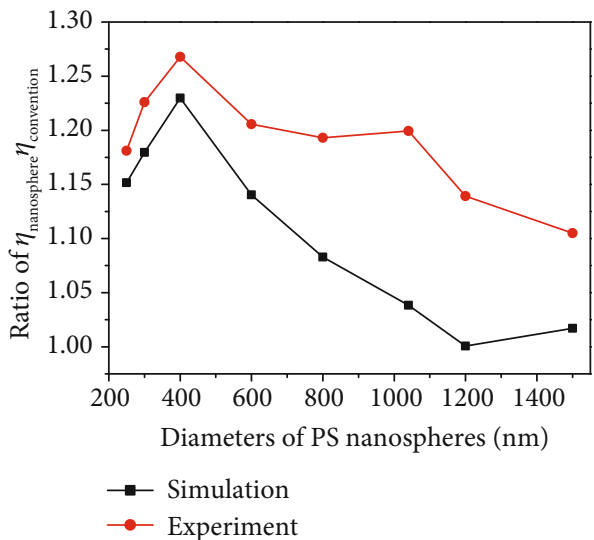

(b)

FIGURE 4: (a) Schematic illustration of the LED simulation model. (b) Dependence of the experimental and simulated enhancement ratios of light extraction at an emission wavelength of $385 \mathrm{~nm}$ on PS nanosphere diameter.

intensity with an incident angle of $23^{\circ}$ showed that the light could be propagated to air through the PS nanospheres. Therefore, it is generally believed that the transmission peak at about $23^{\circ}$ originates from the diffraction of the evanescent field near the PS-ITO interface caused by the guide modes, which is similar to the light extraction behavior of a typical two-dimensional (2D) photonic crystal [19, 32]. However, the distribution of electric field intensity with an incident angle of $31^{\circ}$ shows that the light can be extracted to a PS nanosphere and then exhibits strong confinement inside the PS nanosphere. It is believed that the peak at about $31^{\circ}$ is strongly correlated with the whispering-gallery modes (WGMs) caused by the Mie resonance of individual nanospheres [33, 34]. Furthermore, the WGMs can propagate in the plane of the monolayer of spheres as a dielectric waveguide, as shown in Figure 5(d). Similar behavior has been reported in Ref. [35]. Therefore, the incident light with a certain angle beyond the critical angle can be coupled into the array of nanospheres to form the WGMs. The WGMs would subsequently be diffracted into the far field by the periodic structure as leaky modes. Thus, the natural characteristics of the nanosphere array provide an additional physical 


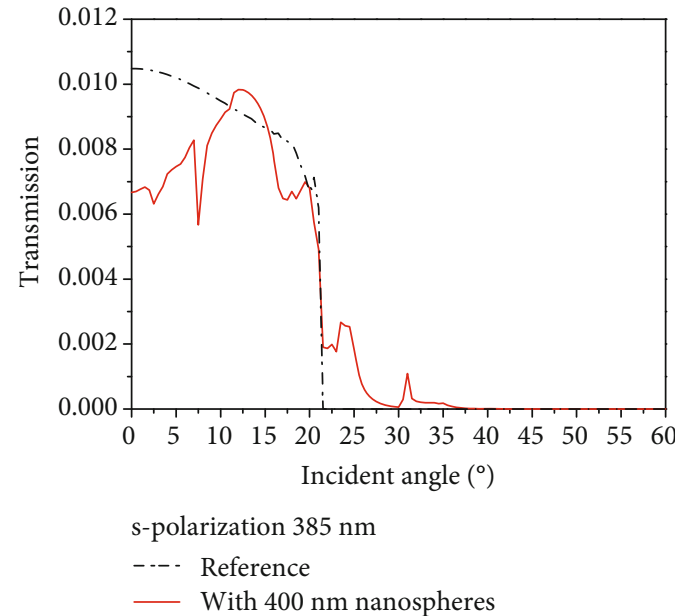

(a)

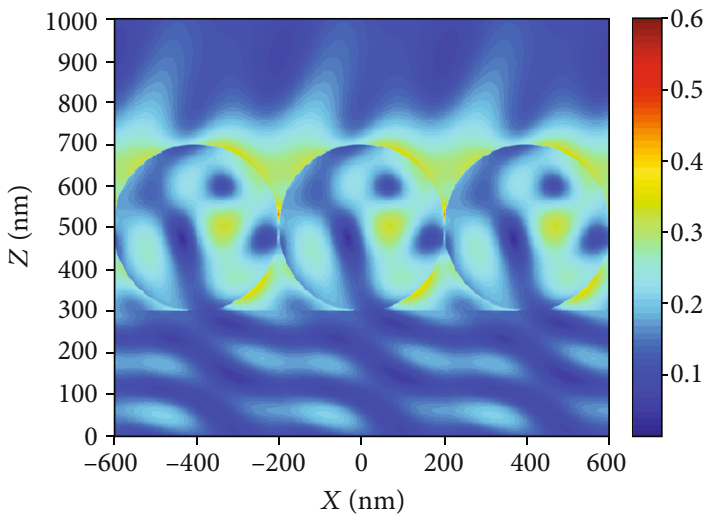

(c)

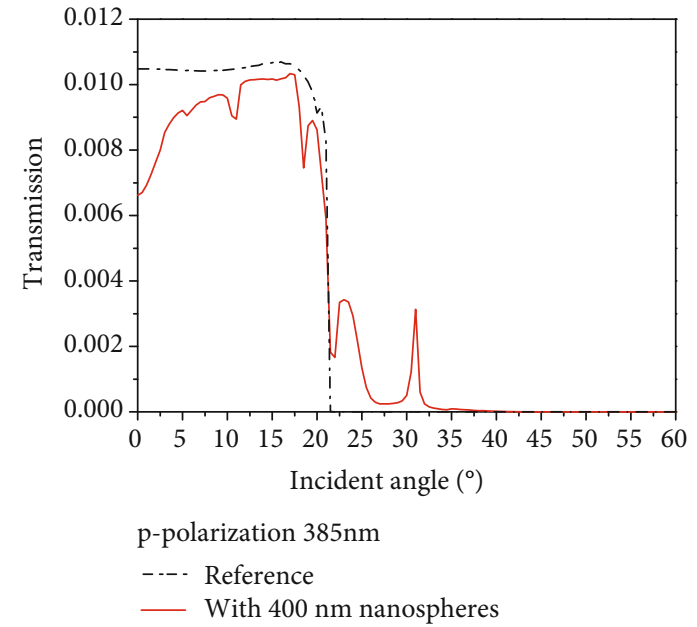

(b)

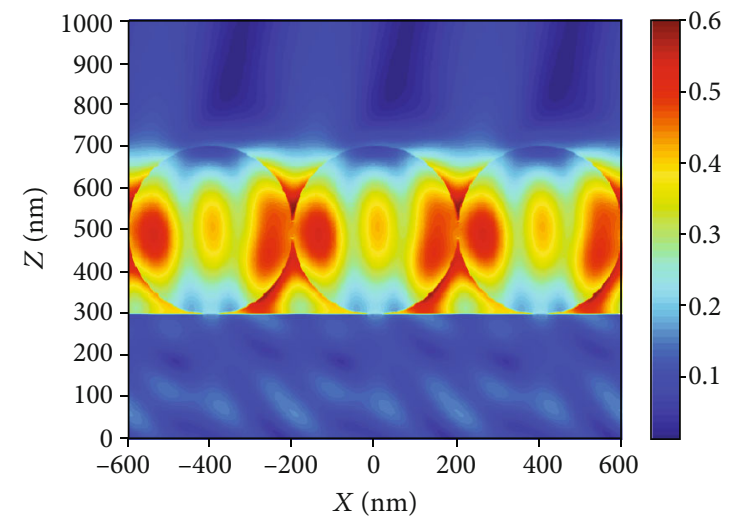

(d)

FIGURE 5: The simulated relationships between transmission and incident angles at a wavelength of $385 \mathrm{~nm}$ for LEDs with and without a monolayer of PS nanospheres with a diameter of $400 \mathrm{~nm}$ under (a) s and (b) p polarizations. Simulations of the spatial distributions of electric field intensity for LEDs covered with a monolayer of PS nanosphere under p polarization at incident angles of (c) $23^{\circ}$ and (d) $31^{\circ}$.

mechanism that increases light extraction. Additionally, several dips below the critical angle appeared in the transmission spectra. These dips resulted in some extra light being diffracted back into the LED. The back-diffracted light can be reflected by the back of the LED and then be re-extracted by the structures, which could raise the ultimate extraction efficiency [19].

The increase of the LEE of the NUV LEDs with a monolayer of hexagonal close-packed PS nanospheres is mainly caused by the following reasons. First, the periodic PS nanosphere arrays can act as $2 \mathrm{D}$ diffraction gratings to take out some guide modes that then propagate inside the LEDs through the near-field coupling. Second, when the size of the PS nanospheres is close to the emission wavelength, the guide modes can be coupled to the spheres with the evanescent field and diffracted into the far field by the periodic structure.

\section{Conclusions}

Monolayers of hexagonal close-packed PS nanospheres with different diameters were prepared on the ITO layer of NUV LEDs. The effect of PS nanospheres on the LEE of LEDs was investigated. The results showed that a PS nanosphere monolayer on the transparent ITO electrode enhanced the NUV optical output of the LED. The maximum LEE was obtained when the nanosphere size was similar to the wavelength of light. Compared with that of the corresponding conventional LED without a PS nanosphere monolayer, the NUV LED with a monolayer of nanospheres of the optimum size resulted in an enhancement of LEE of 1.27-fold at $I=$ $100 \mathrm{~mA}$. The results of FDTD simulations and experiments agreed well. The calculated transmission spectrum showed that part of the incident light beyond the critical angle was extracted when the surface of NUV LEDs was covered with monolayer of PS nanospheres, leading to enhanced LEE. This approach using PS nanospheres has the advantages of low cost and high precision and is suitable for large-area preparation on LEDs. Thus, this is a simple and cost-effective approach to raise the efficiency of NUV LEDs.

\section{Data Availability}

The data used to support the findings of this study are available from the corresponding author upon request. 


\section{Conflicts of Interest}

The authors declare that there is no conflict of interests.

\section{Acknowledgments}

We thank Prof. Zhang-Kai Zhou from Sun Yat-sen University for helpful discussions. This work was partly supported by the National Natural Science Foundation of China (Grant No. 61475038), Science and Technology Program of Guangzhou, China (Grant No. 201804010175), and Special Innovation Project of Department of Education of Guangdong Province (Grant No. 2018KTSCX123).

\section{References}

[1] Y. Muramoto, M. Kimura, and S. Nouda, "Development and future of ultraviolet light-emitting diodes: UV-LED will replace the UV lamp," Semiconductor Science and Technology, vol. 29, no. 8, article 084004, 2014.

[2] M. S. Alias, M. Tangi, J. A. Holguín-Lerma et al., "Review of nanophotonics approaches using nanostructures and nanofabrication for III-nitrides ultraviolet-photonic devices," Journal of Nanophotonics, vol. 12, no. 4, article 043508, 2018.

[3] H. Kim, D. H. Kim, and T. Y. Seong, "Cr/ITO semi-transparent n-type electrode for high-efficiency AlGaN/InGaN- based near ultraviolet light-emitting diodes," Superlattices and Microstructures, vol. 111, pp. 872-877, 2017.

[4] T. H. Seo, S. J. Chae, B. K. Kim, G. Shin, Y. H. Lee, and E. K. Suh, "Enhanced light output power of near-ultraviolet lightemitting diodes with Au-doped graphene for transparent and current-spreading electrode," Applied Physics Express, vol. 5, no. 11, article 115101, 2012.

[5] K. H. Lee, H. J. Park, S. H. Kim et al., "Light-extraction efficiency control in AlGaN-based deep-ultraviolet flip-chip light-emitting diodes: a comparison to InGaN-based visible flip-chip light-emitting diodes," Optics Express, vol. 23, no. 16, article 20340, 2015.

[6] K. R. Son, B. R. Lee, M. H. Jang, H. C. Park, Y. H. Cho, and T. G. Kim, "Enhanced light emission from AlGaN/GaN multiple quantum wells using the localized surface plasmon effect by aluminum nanoring patterns," Photonics Research, vol. 6, no. 1, p. 30, 2017.

[7] S. H. Hong, C. Y. Cho, S. J. Lee et al., "Localized surface plasmon-enhanced near-ultraviolet emission from InGaN/GaN light-emitting diodes using silver and platinum nanoparticles," Optics Express, vol. 21, no. 3, pp. 3138-3144, 2013.

[8] S. H. Hong, J. J. Kim, J. W. Kang et al., "Enhanced optical output of InGaN/GaN near-ultraviolet light-emitting diodes by localized surface plasmon of colloidal silver nanoparticles," Nanotechnology, vol. 26, no. 38, article 385204, 2015.

[9] K. C. Shen, W. Y. Lin, H. Y. Lin, K. Y. Chen, and D. S. Wuu, "Self-textured oxide structure for improved performance of $365 \mathrm{~nm}$ ultraviolet vertical-type light-emitting diodes," Optics Express, vol. 22, no. 15, pp. 17600-17606, 2014.

[10] D. S. Wang, K. X. Zhang, H. W. Liang et al., "Enhanced output power of near-ultraviolet light-emitting diodes by $\mathrm{p}-\mathrm{GaN}$ micro-rods," Chinese Physics Letters, vol. 31, no. 2, article 027101, 2014.
[11] F. H. Fan, Z. Y. Syu, C. J. Wu et al., "Ultraviolet GaN lightemitting diodes with porous-AlGaN reflectors," Scientific Reports, vol. 7, no. 1, p. 4968, 2017.

[12] J. Xu, W. Zhang, M. Peng, J. N. Dai, and C. Q. Chen, "Enhanced luminescence of InGaN-based $395 \mathrm{~nm}$ flip-chip near-ultraviolet light-emitting diodes with $\mathrm{Al}$ as $\mathrm{N}$-electrode," Chinese Physics Letters, vol. 34, no. 7, article 074210, 2017.

[13] H. Jeong, R. Salas-Montiel, G. Lerondel, and M. S. Jeong, "Indium gallium nitride-based ultraviolet, blue, and green light-emitting diodes functionalized with shallow periodic hole patterns," Scientific Reports, vol. 7, no. 1, article 45726, 2017.

[14] J. S. Park, J. H. Kim, J. Y. Na et al., “Ag nanowire-based electrodes for improving the output power of ultraviolet AlGaNbased light-emitting diodes," Journal of Alloys and Compounds, vol. 703, pp. 198-203, 2017.

[15] J. Y. Kim, J. S. Park, J. Y. Na, S. K. Kim, D. Kang, and T. Y. Seong, "Using agglomerated Ag grid to improve the light output of near ultraviolet AlGaN-based light-emitting diode," Microelectronic Engineering, vol. 169, pp. 29-33, 2017.

[16] T. H. Seo, S. Kim, M. J. Kim, H. Kim, and E. K. Suh, "Compound Ag nanocluster-graphene electrodes as transparent and current spreading electrodes for improved light output power in near-ultraviolet light emitting diodes," Journal of Physics D: Applied Physics, vol. 47, no. 21, article 215103, 2014.

[17] T. H. Seo, A. H. Park, S. Park et al., "Improving the graphene electrode performance in ultra-violet light emitting diode using silver nanowire networks," Optical Materials Express, vol. 5, no. 2, p. 314, 2015.

[18] J. Xu, W. Zhang, M. Peng, J. Dai, and C. Chen, "Lightextraction enhancement of GaN-based $395 \mathrm{~nm}$ flip-chip light-emitting diodes by an Al-doped ITO transparent conductive electrode," Optics Letters, vol. 43, no. 11, pp. 26842687, 2018.

[19] Z. Zhu, B. Liu, C. Cheng et al., "Broadband light output enhancement for scintillator using whispering-gallery modes in nanospheres," Physica Status Solidi A: Applications and Materials Science, vol. 211, no. 7, pp. 1583-1588, 2014.

[20] P. Mao, A. Mahapatra, J. Chen, M. Chen, G. Wang, and M. Han, "Fabrication of polystyrene/ZnO micronano hierarchical structure applied for light extraction of light-emitting devices," ACS Applied Materials \& Interfaces, vol. 7, article 19179, 2015.

[21] H. K. Lee, Y. H. Ko, G. S. R. Raju, and J. S. Yu, "Light-extraction enhancement and directional emission control of GaNbased LEDs by self-assembled monolayer of silica spheres," Optics Express, vol. 20, no. 22, pp. 25058-25063, 2012.

[22] P. Zhu and N. Tansu, "Effect of packing density and packing geometry on light extraction of III-nitride light-emitting diodes with microsphere arrays," Photonics Research, vol. 3, no. 4, p. 184, 2015.

[23] P. Zhu, G. Liu, J. Zhang, and N. Tansu, "FDTD analysis on extraction efficiency of GaN light-emitting diodes with microsphere arrays," Journal of Display Technology, vol. 9, no. 5, pp. 317-323, 2013.

[24] X. H. Li, R. Song, Y. K. Ee, P. Kumnorkaew, J. F. Gilchrist, and $\mathrm{N}$. Tansu, "Light extraction efficiency and radiation patterns of III-nitride light-emitting diodes with colloidal microlens arrays with various aspect ratios," IEEE Photonics Journal, vol. 3, no. 3, pp. 489-499, 2011.

[25] Y. K. Ee, P. Kumnorkaew, R. A. Arif et al., "Optimization of light extraction efficiency of III-nitride LEDs with self-assembled 
colloidal-based microlenses," IEEE Journal of Selected Topics in Quantum Electronics, vol. 15, no. 4, pp. 1218-1225, 2009.

[26] X. H. Li, P. Zhu, G. Liu et al., "Light Extraction Efficiency Enhancement of III-Nitride Light-Emitting Diodes by Using 2-D Close-Packed TiO2 12 Microsphere Arrays," Journal of Display Technology, vol. 9, no. 5, pp. 324-332, 2013.

[27] C. Y. Fang, Y. L. Liu, Y. C. Lee, H. L. Chen, D. H. Wan, and C. C. Yu, "Nanoparticle stacks with graded refractive indices enhance the omnidirectional light harvesting of solar cells and the light extraction of light-emitting diodes," Advanced Functional Materials, vol. 23, no. 11, pp. 1412-1421, 2013.

[28] Z. Chen, W. J. Liu, W. Wei, G. Y. Chen, B. Zhang, and C. Jin, "Improving the extraction efficiency of planar GaN-based blue light-emitting diodes via optimizing indium tin oxide nanodisc arrays," Journal of Display Technology, vol. 12, p. 1, 2016.

[29] X. Wang, C. J. Summers, and Z. L. Wang, "Large-scale Hexagonal-Patterned growth of aligned $\mathrm{ZnO}$ nanorods for nano-optoelectronics and nanosensor arrays," Nano Letters, vol. 4, no. 3, pp. 423-426, 2004.

[30] B. Deul Ryu, P. Uthirakumar, J. Hye Kang et al., "Enhanced light output power of GaN-based light-emitting diodes by nano-rough indium tin oxide film using $\mathrm{ZnO}$ nanoparticles," Journal of Applied Physics, vol. 109, no. 9, article 093116, 2011.

[31] Z. Zhu, B. Liu, C. Cheng, Y. Yi, H. Chen, and M. Gu, "Improved light extraction efficiency of cerium-doped lutetium-yttrium oxyorthosilicate scintillator by monolayers of periodic arrays of polystyrene spheres," Applied Physics Letters, vol. 102, no. 7, article 071909, 2013.

[32] A. Knapitsch, E. Auffray, C. W. Fabjan et al., "Results of photonic crystal enhanced light extraction on heavy inorganic scintillators," IEEE Transactions on Nuclear Science, vol. 59, no. 5, pp. 2334-2339, 2012.

[33] M. López-García, J. F. Galisteo-López, C. López, and A. García-Martín, "Light confinement by two-dimensional arrays of dielectric spheres," Physical Review B, vol. 85, no. 23, article 235145, 2012.

[34] H. T. Miyazaki, H. Miyazaki, K. Ohtaka, and T. Sato, "Photonic band in two-dimensional lattices of micrometer-sized spheres mechanically arranged under a scanning electron microscope," Journal of Applied Physics, vol. 87, no. 10, pp. 7152-7158, 2000.

[35] B. M. Möller, U. Woggon, and M. V. Artemyev, "Coupled-resonator optical waveguides doped with nanocrystals," Optics Letters, vol. 30, no. 16, pp. 2116-2118, 2005. 